\title{
Análisis del comportamiento de compra respecto a la oferta de productos lácteos en El Oro
}

\author{
Analysis of purchase behavior with respect to the supply of dairy products in \\ Province of El Oro
}

\author{
Villavicencio Ma. Fernanda \\ Universidad Técnica de Machala \\ mvillavicencio@utmachala.edu.ec \\ Machala - Ecuador \\ Macas Fernando \\ Universidad Técnica de Machala \\ cfmacas_est@utmachala.edu.ec \\ Machala - Ecuador \\ Jaramillo Marco \\ Universidad Técnica de Machala \\ mjaramill5@utmachala.edu.ec \\ Machala - Ecuador \\ Correa Génesis \\ Universidad Técnica de Machala \\ gcorrea3@utmachala.edu.ec \\ Machala - Ecuador
}

\section{Resumen}

Analizar los factores que influyen en el comportamiento de compra del consumidor de productos lácteos zarumeños en la provincia de El Oro. Se realizó un estudio cuantitativo, empleando muestreo probabilístico estratificado para inferir sobre la población en estudio, en este caso, las familias de los cantones de la parte bajan de la provincia de El Oro, abarcando una muestra de 383 personas para una población de 166.768 familias. Los datos se procesaron y analizaron en SPSS $®$. Los resultados del análisis muestran que el porcentaje de consumo de lácteos zarumeños en los cantones objeto de estudio es el $54.20 \%$, los consumidores en su mayoría pertenecen al cantón Machala; presentan además una frecuencia de compra semanal en mercados y tiendas de barrio durante los fines de semana; las razones principales de su elección, es el gusto por los mismos y costumbres; de la oferta de productos, el preferido por los consumidores es el queso zarumeño. Se evidencia también, que las principales fuentes de información consideradas, para la decisión de compra, son las sugerencias de familiares y amigos, presentando bajos porcentajes en medios de comunicación tradicionales. Los resultados de este estudio pueden proporcionar ciertas bases para entender el comportamiento 
de compra del consumidor orense en referencia a productos lácteos elaborados en el cantón Zaruma, presentando importantes implicaciones para los productores, con el fin de que se desarrollen adecuadas estrategias de comercialización y distribución para los cantones de la parte baja de la provincia.

Palabras clave: comportamiento de compra, comportamiento del consumidor, productos lácteos, hábitos de consumo, lácteos zarumeños.

\begin{abstract}
To analyze the factors that influence the consumer's purchasing behavior of Zarumeño dairy products in the province of El Oro. A quantitative study was conducted, using stratified probability sampling to infer about the population under study, in this case, the families of the districts of the lower part of the province of El Oro, covering a sample of 383 people for a population of 166,768 families. The data were processed and analyzed in SPSS®. The results of the analysis show that the percentage of consumption of Zarumeño dairy products in the districts under study is $54.20 \%$, the majority of consumers belong to the Machala district; they also have a weekly purchase frequency in markets and neighborhood stores on weekends; the main reasons for their choice are taste and customs; of the products offered, the favorite of consumers is Zarumeño cheese. It is also evidenced, that the main sources of information considered, for the purchase decision, are the suggestions of family and friends, presenting low percentages in traditional media. The results of this study can provide certain bases for understanding the purchasing behavior of the Orense consumer in reference to dairy products produced in the Zaruma district, presenting important implications for producers, so that adequate marketing and distribution strategies can be developed for the districts of the lower part of the province.
\end{abstract}

Keywords: purchase behavior, consumer behavior, dairy products, consumption habits, dairy products from Zaruma.

\title{
Introducción
}

El comportamiento del consumidor protagoniza un rol esencial en la relación directa entre el ofertante y el demandante, entiéndase como ofertante aquella persona o institución que pretende satisfacer una necesidad a través de un producto, servicio o incluso idea (Salgado Beltrán et al., 2017); por otro lado, el demandante como aquella persona o institución que carece de algo. Es así, necesario que las empresas, ya sean estas pequeñas, medianas, nacionales e internacionales, conozcan al consumidor (Encarnación, 2019).

Como menciona Duran Meléndez (2016), el entorno globalizado, cambiante y altamente competitivo, requiere estar pendiente a las exigencias del entorno, cumplir sus expectativas, a través de los estudios de la conducta del consumidor, responder a preguntas frecuentes, pero altamente relevantes como: ¿Quién compra?, ¿Por qué ?, ¿Cuánto compra?, ¿Dónde compra?, sus gustos y preferencias, entre otros. De esta manera logra una empresa producir para el consumidor, generar valor y una experiencia diferenciadora ante la competencia para sobrevivir en el mercado.

Es entonces inminente el cambio constante que experimenta el entorno, ya que las personas cada vez cambian sus pensamientos, sus gustos, sus exigencias, sus sentimientos y los hábitos de consumo. Huanga León (2019), expresa que el consumidor tras esa evolución se vuelve más 
selectivo y exigente al momento de llevar a cabo una compra, buscando minimizar el tiempo, esfuerzo y recursos invertidos obteniendo el mejor beneficio. (Hershkovitz1, 2017).

La importancia de esta investigación, se centra en la necesidad de analizar el comportamiento de compra del consumidor Orense respecto a la oferta de productos lácteos elaborados artesanalmente en el cantón Zaruma. Según los autores Upadhyay, Banerjee \& Panigrahi (2020) en la actualidad se encuentran estudios nacionales, sin embargo, existe carencia de investigaciones actuales y sobre todo del entorno seleccionado.

Las empresas locales de cualquier tamaño, a través de la resolución de la presente problemática, se verán beneficiadas al tener una guía sobre el comportamiento de compra del consumidor en este sector productivo. Así lo expresa Campoverde Vega (2018), quien enfatiza la importancia de conocer el comportamiento del consumidor con el propósito de elaborar estrategias y tácticas ideales para el beneficio de ambas partes, la empresa y la satisfacción del cliente.

De acuerdo a los autores Almeida \& Ureña (2019), la industria de lácteos ocupa el tercer lugar dentro de la categoría de alimentos, esto se da debido al incremento de la capacidad adquisitiva, que mejora la condición social, provocando en los consumidores requerir de una diversidad en alimentos, es así que se debe aprovechar dicho auge de los productos lácteos y sus derivados.

El objetivo de la investigación consiste en analizar los factores que influyen en el comportamiento de compra del consumidor de productos lácteos zarumeños en la provincia de El Oro. Según Beltrán (2018), conocer el comportamiento de compra se vuelve imprescindible en las empresas, ya que permite crear certeza que el producto está orientado hacia el consumidor, evidenciando que el perfil del cliente potencial esté bien definido y se refleja en las características de los productos que se pretenden ofertar.

La metodología aplicada para el presente análisis, asume resolver las siguientes interrogantes: ¿Las familias de los cantones de la parte baja de la provincia de El Oro, prefieren consumir productos lácteos zarumeños?, ¿Cuál es su frecuencia de compra?,¿Cuáles son los canales de distribución que prefiere el comprador?, ¿Cuáles son las fuentes de información presentes en el proceso de decisión de compra?

\section{Materiales y Métodos}

Para la realización de esta investigación se utilizó el método cuantitativo, inclinándose por la formulación de encuestas. Una vez definida la herramienta de investigación se tomaron en consideración los cantones de la parte baja de la provincia de El Oro, es así que intervienen El Guabo, Machala, Huaquillas, Arenillas, Santa Rosa y Pasaje. Según el (INEC, 2010), la población objetivo a ser considerada dentro de la investigación corresponde a $608^{`} 705$ habitantes; así también el número de hogares por los diferentes cantones de la provincia nos da un total de 166768. Para la determinación del tamaño de la muestra se aplicó la fórmula de población definida, de acuerdo a la población de los cantones objeto de estudio.

$$
\boldsymbol{n}=\frac{Z^{2} * P * Q * N}{E^{2}(N-1)+Z^{2} * P * Q}
$$

Como resultado de ello, se obtuvo una muestra de 383, con un error del $5 \%$ y un nivel de confianza del 95\%. Una vez conocida la muestra, se aplicó la encuesta, la misma que se diseñó con el objetivo de poder conocer, los gustos, preferencias y comportamiento que tienen los consumidores ante los productos lácteos y sus derivados, para ello se utilizó el muestreo aleatorio estratificado, donde los estratos son los diferentes hogares de los cantones anteriormente mencionados, encuestando de esta forma a 1 persona por familia.

Es importante mencionar que las variables a analizar en este artículo, tienen relación con el comportamiento de compra de los productos lácteos zarumeños, más no de lácteos en general, 
es así que del total de la muestra recogida (383), un número de 237 encuestados son considerados datos válidos para el análisis, ya que manifestaron consumir dichos productos específicamente.

Los estadísticos descriptivos se analizaron a través del software Spss IBM, realizando un análisis univariado de las siguientes variables cualitativas nominales: sociodemográficas, consumo, frecuencia de compra, preferencia de canal de distribución, fuente de información utilizada. Además de un análisis bivariado relacionando algunas de las variables mencionadas con el fin de profundizar algunos aspectos del estudio.

\section{Resultados y Discusión}

A continuación, podemos observar los resultados en cuanto a la distribución de la muestra en la Tabla 1. De acuerdo a la pregunta de clasificación de género tenemos que, el $62,7 \%$ de la muestra pertenece al género femenino; la edad de los encuestados se encuentra entre los 16 a 47 años en un 79.4\%; el estado civil casado representa el $42,3 \%$ de la muestra.

La mayoría de los encuestados (55\%) trabajan y el $18 \%$ son amas de casa, dato importante ya que se ha indagado en las personas que por su capacidad adquisitiva o su rol en el hogar toman las decisiones de compra. El $46 \%$ de los encuestados tienen educación secundaria y el $37 \%$ posee un título de tercer nivel. El 51\% de la muestra cuenta con un ingreso mensual entre \$201 y $\$ 602$.

La distribución de la muestra resulta ser adecuada, puesto que incluye rangos de edad, ocupación, nivel de educación e ingreso mensual, característicos de perfil del consumidor potencial del tipo de productos a analizar, es así que, obtenemos información pertinente y que aporta para dar respuesta a las preguntas de investigación planteadas.

Tabla 1.

Distribución de la muestra 


\begin{tabular}{ccc}
\hline Consumidores & Total & \\
\hline Género & $\mathbf{N}^{\mathbf{0}}$ & \\
Masculino & 143 & 37.3 \\
Femenino & 240 & 62.7 \\
Edad & 158 & 41.3 \\
16-31 años & 146 & 38.1 \\
32-47 años & 64 & 16.7 \\
48-63 años & 15 & 3.9 \\
Más de 64 años & 123 & 32.1 \\
Estado civil & 162 & 42.3 \\
Soltero & 22 & 5.7 \\
Casado & 13 & 3.4 \\
Divoriado & 63 & 16.4 \\
Viudo & & \\
Unión libre & 211 & 55.1 \\
Ocupación & 51 & 13.3 \\
Trabaja & 41 & 10.7 \\
Estudia & 69 & 18.0 \\
Trabaja y estudia & 11 & 2.9 \\
Ama de casa & & \\
Sin ocupación & 2 & 0.5 \\
Nivel de estudio & 50 & 13.1 \\
Sin nivel de estudio & 177 & 46.2 \\
Primaria & 144 & 37.6 \\
Secundaria & 10 & 2.6 \\
Tercer nivel & & 23.0 \\
Cuarto Nivel & 88 & 29.0 \\
Ingreso Mensual & 111 & 22.7 \\
\$0 a \$200 & 87 & 15.1 \\
\$201 a \$401 & 58 & 6.8 \\
\$402 a \$602 & 26 & 3.4 \\
\$603 a \$803 & 13 & \\
\$804 a \$1004 & & \\
Más de \$1005 & & \\
\hline & & \\
\hline
\end{tabular}

\section{Preferencia de consumo de lácteos zarumeños}

\section{Consumo lácteos zarumeños}

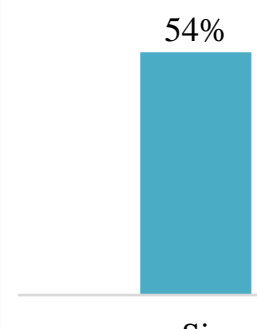

$\mathrm{Si}$

No

Figura 1.

Consumo de lácteos zarumeños

$\mathrm{Al}$ consultar a las familias de los diferentes cantones, acerca de su consumo actual de productos lácteos zarumeños, el 54.20\% respondió afirmativamente. A demás, del total de los encuestados el $62 \%$ manifiesta haber consumido alguna vez estos productos. La preferencia de consumo de los Orenses se debe principalmente, a que dichos productos son de su agrado (65\%) y por costumbre (21\%).De la oferta de los mencionados productos, el queso, tiene un mayor consumo con el $86 \%$, notándose su presencia en la dieta de los habitantes de los cantones mencionados. Podemos mencionar también, que el queso Zarumeño, en la parte baja de la provincia es preferido por su sabor, el cual claramente es un atributo diferenciador de los otros productos de su misma categoría y que está instalado en la percepción del consumidor. 


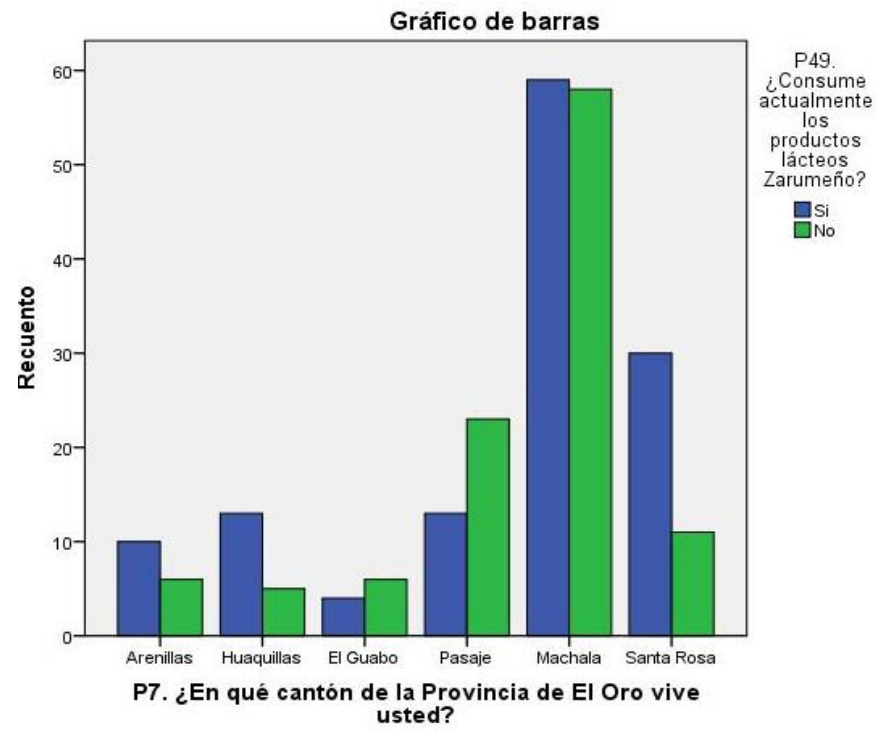

Figura 2.

Consumo de lácteos zarumeños por cantón

Al realizar un análisis bivariado entre cantón de residencia y el consumo actual de dichos productos, podemos observar que el $45.7 \%$ de los consumidores se encuentran en el cantón Machala, seguido por el $23.3 \%$ en el cantón Santa Rosa.

\section{Frecuencia de compra}

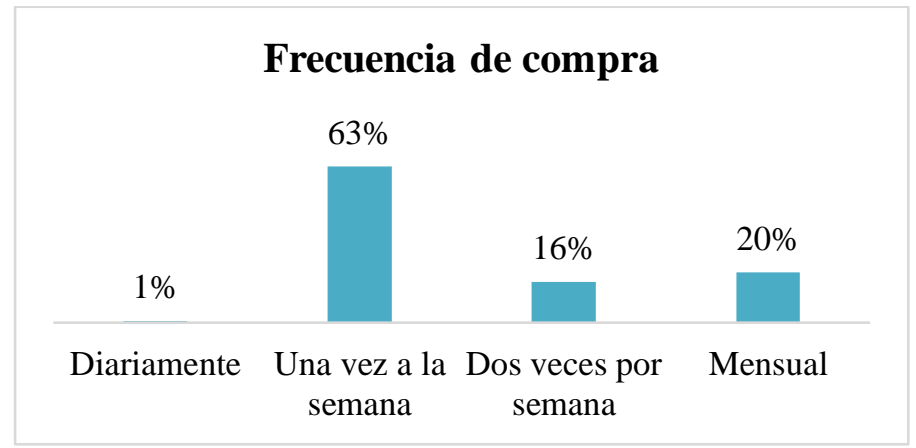

Figura 3.

Frecuencia de compra de productos lácteos Zarumeños

En la figura 3 se puede observar la frecuencia de compra de los productos lácteos, demostrando que son adquiridos una vez a la semana (63\%), siendo los fines de semana los días predilectos para la elección de dichos productos. Generalmente, este tipo de productos mantienen esta frecuencia de compra, por el interés del consumidor de alimentarse con productos frescos; además de aprovechar la variada oferta a la que acceden en los puntos de abastecimiento, situados en los diferentes cantones, durante los fines de semana.

\section{Preferencia de canales de distribución}




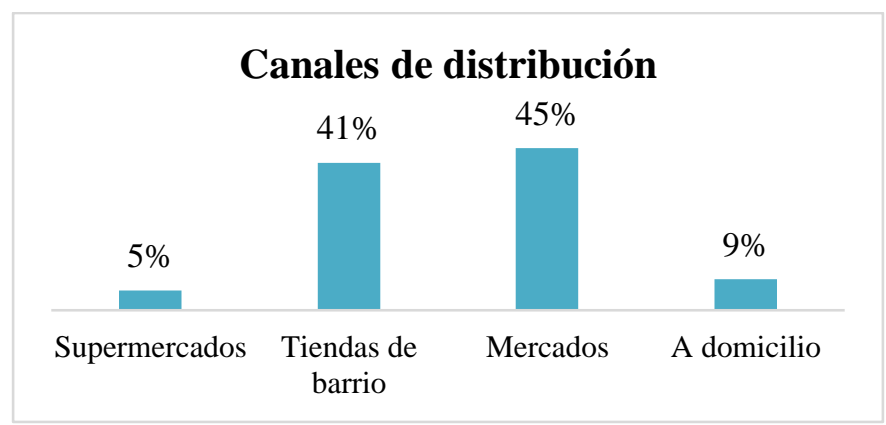

Figura 4.

Canales de distribución.

Los lugares preferidos por los compradores para adquirir los productos lácteos zarumeños son los mercados (45\%) y las tiendas de barrio (41\%), es importante mencionar que, al ser productos elaborados artesanalmente, su comercialización se centra en canales de distribución sin exigencia de registro sanitario. Es importante recalcar que una de las razones más fuertes para no consumir estos productos, es la dificultad de encontrarlos con un (24.3\%).

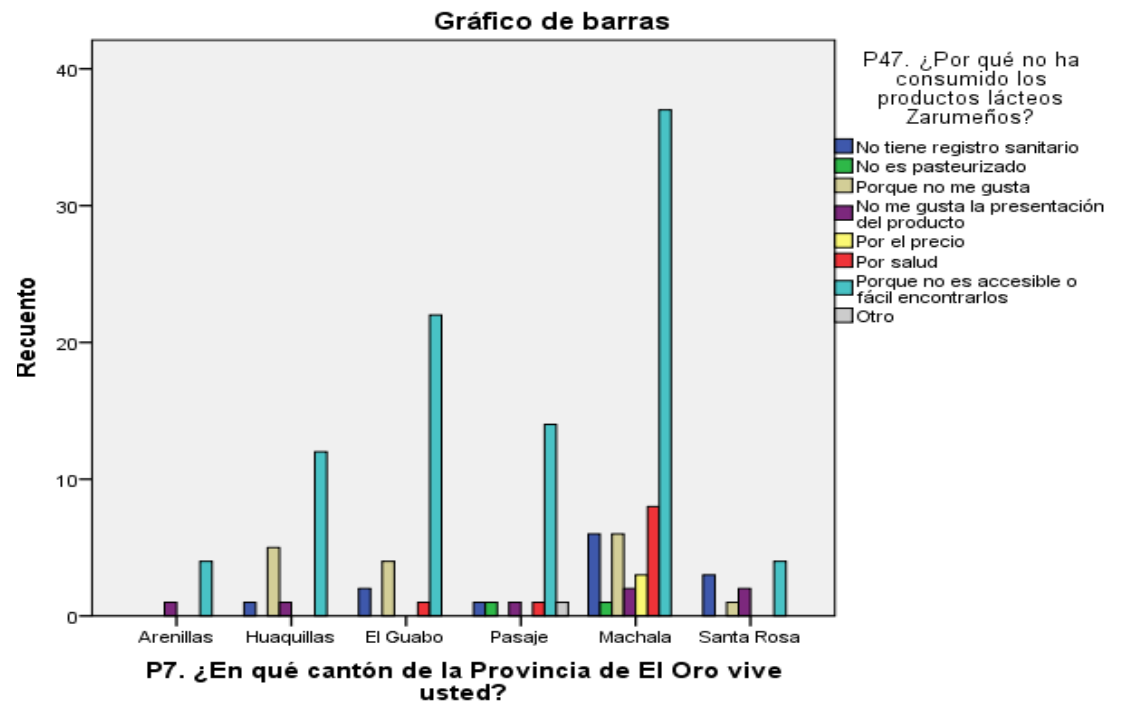

Figura 5.

Motivos de no consumo de productos zarumeños de acuerdo al cantón.

Es importante recalcar que, al analizar las dos variables, cantón de residencia y razón por la que no consume productos lácteos zarumeños, se observa en todos los cantones, que los encuestados afirman haber dejado de consumir dichos productos al no tener fácil acceso a los mismos; evidenciando una necesidad latente de expandir el canal de distribución. 


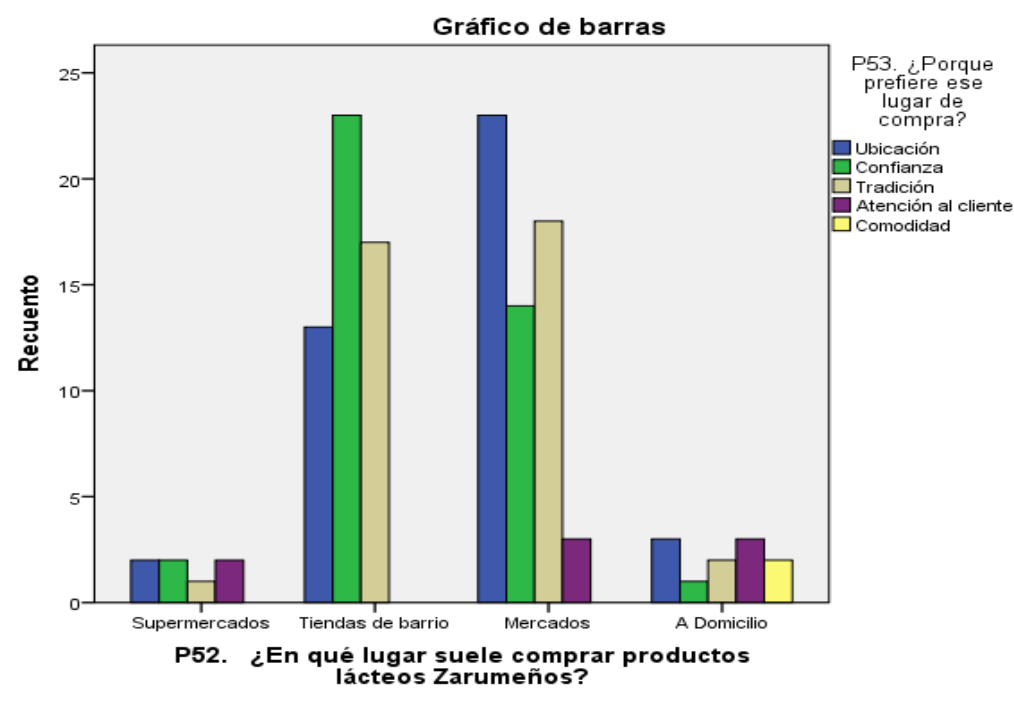

Figura 6.

Motivo de preferencia de lugar de compra.

Al realizar un análisis bivariado entre lugar de compra de preferencia y razón por la que escoge dicho lugar, podemos evidenciar que el $45 \%$ de los encuestados prefieren comprar en mercados, el $17.8 \%$ prefiere estos por su ubicación y un $14 \%$ por tradición; mientras que, en referencia a tiendas de barrio, el $41.1 \%$ de los encuestados prefieren dicho lugar y compran en estas por la confianza un $17.8 \%$ y un $13.2 \%$ por tradición.

\section{Fuentes de información en el proceso de decisión de compra}

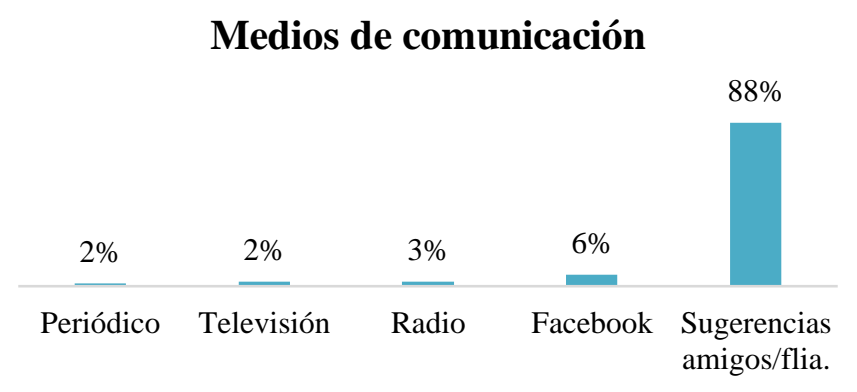

Figura 7.

Medios de comunicación

A pesar de que hoy en día los medios tecnológicos impactan en casi todos los productos y servicios, los productores de lácteos zarumeños no los utilizan para difundir su oferta, es así que los resultados indican, que los encuestados obtienen información de estos, a través de las sugerencias de personas cercanas con un (88\%). A demás manifiestan, que se interesan por las "degustaciones" como estrategia de promoción de dichos productos, lo que sugeriría considerar ciertas tácticas en los puntos de venta.

\section{Conclusiones}


A partir de la presente investigación se ha dado respuesta a las preguntas planteadas a través de fundamentos teóricos necesarios para el objeto de estudio, acorde a ello, podemos concluir que las familias de los cantones de la parte baja de la provincia de El Oro, son potenciales consumidores de productos lácteos elaborados en el cantón Zaruma; ciertamente por factores socioculturales, identificados en el estudio, que son determinantes en el proceso de decisión de compra (Salazar Lozada et al., 2019).

Al analizar el comportamiento de consumo, observamos la decisión y alto interés por adquirir dichos productos que forman parte de la dieta alimenticia del orense, por el hecho de que estos satisfacen sus gustos y preferencias y su consumo es parte de sus costumbres. Es así que, en los resultados observamos, su alta frecuencia de compra sobre todo en el cantón Machala y una clara limitante, como la falta de disponibilidad en los puntos de venta.

Un factor importante en la decisión de compra del consumidor, son las fuentes de información; al ser la oferta de productos lácteos de Zaruma netamente artesanal y local, no tienen presencia en medios de comunicación masiva, es así que la información acerca de los mismos, los consumidores la encuentran a través de recomendaciones familiares y de amistades. Sin embargo, una opción de difusión de la oferta se podría realizar a través de las redes sociales que tienen un acelerado posicionamiento en el consumo de medios digitales y que representan aún un menor costo para el productor.

Los resultados forman parte de un estudio llevado a cabo en una zona específica, sin lugar a duda abre la puerta a futuras investigaciones en otros mercados y elaborar propuestas o proyectos enfocados en la comercialización y distribución más formal de los lácteos Zarumeños, tomando como referencia la acogida en los cantones donde se llevó a cabo el estudio.

\section{Referencias}

Almeida, R., \& Ureña, F. (2019). Lealtad de marca de empresas de telefonía móvil en los estudiantes de la Universidad Católica Boliviana "San Pablo" Unidad Académica Cochabamba. Revista Perspectivas.

Beltrán, L. S. (2018). Segmentation of organic food consumers based on their attitudes, values and environmental beliefs. Contaduría y Administración, 21.

Campoverde Vega, M. X. (2018). Comportamiento del consumidor para evaluar la factibilidad de expansión de negocio. (Examen Complexivo). Universidad Técnica de Machala, Machala.

Durán Meléndez, E. (2016). Estudio del consumo de leche y sus derivados en el Municipio de Oaxaca de Juarez, México. Revista Mexicana de Agronegocios, 442.

Encarnación, E. T. (2019). Análisis del comportamiento del consumidor de la Hacienda. UNIVERSIDAD CATÓLICA DE SANTIAGO DE GUAYAQUIL.

Hershkovitz1, S. (2017). BNot Buying Cottage Cheese^ ${ }^{\wedge}$ Motivations for Consumer Protestthe Case of the 2011 Protest in Israel. CrossMark, 12.

Huanga León, V. E. (2019). Análisis del comportamiento de los consumidores en la industria del celular en la Ciudad de Machala. (Examen complexivo). Universidad Técnica de Machala, Machala. 
INEC. (2010). Obtenido de Población y Demografía Del Ecuador: https://www.ecuadorencifras.gob.ec/censo-de-poblacion-y-vivienda/

Salazar Lozada, G. M., García Rodríguez, J. F., \& Méndez Olán, L. A. (2019). ANÁLISIS DEL PATRÓN DE COMPORTAMIENTO DEL CONSUMIDOR. Original, 18.

Salgado Beltrán, L., Beltrán Morales, L. F., \& Velarde Mendivil, A. T. (2017). Attitudes and Sensory Perceptions of Food Consumers towards Technological Innovation in Mexico: A Case-Study on Rice-Based Dessert. Sustainability, 15.

Upadhyay, S., Banerjee, A., \& Panigrahi, P. K. (2020). Causal evolution of global crisis in financial networks. ELSEVIER, 10. 